\title{
Unexpected fast and massive release of minor and trace elements during silicate/water interactions
}

\author{
DAMIEN LEMARCHAND*', THIERRY PERRONE', \\ RENE BOUTIN', REMY SAINT-LYS', DAMIEN DAVAL \\ LHyGeS, CNRS/University of Strasbourg, France. \\ (*damien.lemarchand@unistra.fr)
}

The chemical mobility of minor and trace elements during silicate/water interactions has many economical implications either being exploited as ore mining or, to the opposite, leading to toxic accumulation in the environement. The relative release rate of trace elements compared to major ones during silicate/water interactions is generally interpreted in the frame of their distribution in silicate minerals being either i) randomly distributed in the crystal lattice; ii) zoned and reflecting the crystal grotwh history or iii) associated to mineral inclusions. In all cases, the release of trace elements is considered as resulting from the dissolution of the mineral surface in contact with the bulk fluid. Recent works, mostly focused on carbonates, however tend to indicate that the pre-exisitng or development of nanoporosity may lead to anomalous release of trace elements by allowing a larger than expected volume of the crystal to be exposed to water.

In this study, we conducted a series of experiments to determine and compare the release rates of minor and trace elements during albite/water interactions. The experimental setups allow to monitor the release of minor and many trace elements during total dissolution in an acidic and hot fluid as well as surface ionic exchange with $\mathrm{NH}_{4} \mathrm{Cl}$ at room temperature, i.e. without dissolution. In dissolution experiments, our observations demonstrate a rapid and massive release of almost all minor and trace elements compared to major ones, inidicating an heterogeneous distribution of these elements in the crushed albite crystals and their facilited accessibility to water. Careful examination of individual release rates clearly demonstrates the importance of each element chemical property and rules out the hypothess of chemical zoning or significative contribution of mineral impurities. Same results, but in much smaller extent, are obtained during surface exchange reactions indicating that the fast release of trace elements is not necessarily related to dissolution processes and/or the development of nanopores.

These results directly ask the question of the transport processes including the structural properties of the mineral lattice and the chemical composition of the percolating fluid. 